\title{
Inverted circadian variation of arterial pressure in a geriatric patient: an indicator of autonomic dysfunction
}

\author{
Siddhartha Lieten ${ }^{1,2^{*}}$, Aziz Debain ${ }^{1,2}$, Bert Bravenboer ${ }^{1,2,3}$ and Tony Mets ${ }^{1,2}$
}

\begin{abstract}
Background: Orthostatic hypotension $(\mathrm{OH})$ in geriatric patients frequently involves a component of autonomic failure (AF). The combination of $\mathrm{OH}$ with nocturnal hypertension (NHT) is indicative of AF, which is described as pure (PAF), when neurologic symptoms are absent, or as multisystem atrophy (MSA), when combined with motor disturbance (Parkinsonism or Parkinson disease).

Case presentation: An 87-year-old man presented with long-lasting $\mathrm{OH}$. He frequently fell, causing several fractures, and he developed heart failure. Blood pressure (BP) registration revealed a reversal of the day-night rhythm with NHT. An 18-FDG PET brain CT scan showed cerebellar hypometabolism, indicating MSA.

Conclusions: This case demonstrates the use of continuous BP registration in geriatric patients with $\mathrm{OH}$ for diagnosing NHT. It illustrates the usefulness of 18-FDG PET brain CT scan to specify the nature of the AF. The case also illustrates the difficulty of managing the combination of $\mathrm{OH}$ and $\mathrm{NHT}$.
\end{abstract}

Keywords: Orthostatic hypotension, Nocturnal hypertension, Autonomic failure, 18-FDG PET brain CT scan

\section{Background}

Syncopes and falls are major geriatric issues, often related to blood pressure (BP) abnormalities. Orthostatic hypotension $(\mathrm{OH})$ is a frequent finding in older people with a prevalence of more than $20 \%$ above the age of 75 years $[1,2]$. The origin of $\mathrm{OH}$ is complex, involving normal aging-related physiological changes, diseases, and drug induced etiologies.

Aging in itself results in a tendency towards $\mathrm{OH}$ by decreased cardiac output, bradycardia, and decreased baroreceptor sensitivity [3]. Postprandial intestinal vascular dilatation will induce peripheral blood pooling, as will the

\footnotetext{
* Correspondence: Siddhartha.Lieten@uzbrussel.be

'Department of Geriatrics, Universitair Ziekenhuis Brussel, Laarbeeklaan 101, B-1090 Brussels, Belgium

${ }^{2}$ Frailty in Aging (FRIA) investigation group, Faculty of Medicine \& Pharmacy, Vrije Universiteit Brussel (VUB), Laarbeeklaan 103, B-1090 Brussels, Belgium

Full list of author information is available at the end of the article
}

tendency for decreased muscular activity in old age. Subsequently, both will contribute to $\mathrm{OH}[3,4]$.

The tendency for $\mathrm{OH}$ is often aggravated by pathological changes, such as anemia, common cardiac abnormalities (aortic valve stenosis, arrhythmias), diabetes mellitus (particularly in the postprandial phase after a carbohydrate rich meal), or changes resulting in a reduced circulating volume (e.g. adrenal failure). In addition, medication that can either produce hypovolemia (diuretics) or vasodilatation (anti-hypertensives, psychotropics, alpha-lytic drugs for prostatic disease) [5-7] frequently can provoke $\mathrm{OH}$.

A component of autonomic failure (AF) is involved in many older patients with $\mathrm{OH}$. It was observed in $27 \%$ of a group of 100 patients referred for $\mathrm{OH}$ [5]. If no other neurologic symptoms are present, the condition is designated as Pure Autonomic Failure (PAF); if accompanied by motor disturbances, it is referred to as MultipleSystem Atrophy (MSA). The motor component in MSA, described as "Parkinsonism (bradykinesia with rigidity or 
tremor or both), usually with a poor or unsustained motor response to chronic levodopa therapy" can be present in a various degrees [8]. Brain autonomic function is related to the activity of the dopaminergic system, which is known to decline with aging [9]. In recent animal experiments, epigenetic factors, which increase with aging, appear to be responsible for this decline [10]. In this context, it is not surprising that AF occurs more frequently in synucleopathies, the group of neurodegenerative diseases, consisting of Parkinson's disease (PD) and Lewy body dementia [11].

$\mathrm{AF}$ is also observed in peripheral neuropathies. These become more frequent with aging and are mainly seen in the context of diabetes mellitus, chronic kidney disease, and vitamin B12 deficiency [12-14].

The concomitant presence of nocturnal hypertension (NHT) can complicate the management of $\mathrm{OH}$. It is well known that BP has a circadian variation, with higher daytime and lower nighttime values, which drop on average by $10-20 \%$ [15]. Some patients with $\mathrm{OH}$ can present NHT, often described as "non-dipping" or "reverse dipping" [16, 17]. At the origin of this NHT is the AF, resulting in insufficient sympathetic output while standing, but exerting residual activity in supine position $[18,19]$. In a group of $52 \mathrm{OH}$ patients (average age 77 years), NHT was present in $71 \%$ of them [20]. The association between $\mathrm{OH}$ and NHT can be found in PD patients. In $\mathrm{PD}$ patients with $\mathrm{OH}$, non-dipping was observed more frequently ( $78 \%$ vs. $67 \%$ in de non-OH patients) [21]. In another group of $188 \mathrm{PD}$ patients, $\mathrm{OH}$ was observed frequently $(30 \%)$, while NHT was present in $25 \%$ of them and both were associated [22].

Loss of a normal BP pattern with NHT is thought to contribute to the development of cardiovascular disease (CVD), chronic kidney disease, cognitive decline, and dementia [23-28]. Therefore, improved awareness of a non-dipping pattern of BP is important.

Here we describe a patient with multiple disorders, who illustrates the complex situation of $\mathrm{OH}$ combined with NHT.

\section{Case presentation}

An 87-year-old man (weight $80.9 \mathrm{~kg}$, height $1.79 \mathrm{~m}$ and BMI $25.3 \mathrm{~kg} / \mathrm{m}^{2}$ ) presented with a syncope and fall at the emergency department. Upon arrival (at 4 p.m.) he was well oriented; BP (seated) was $168 / 59 \mathrm{mmHg}$ with a regular pulse at $65 / \mathrm{min}$ (auscultatory). He had dyspnea (respiratory rate $15 / \mathrm{min}$ ) with an arterial $\mathrm{O}_{2}$ saturation of $89 \%$ (98\% after oxygen administration). His mouth mucosa was dry and he had bilateral pulmonary stasis, but no jugular tumescence. There was a slight degree of peripheral pitting edema and he had a urethral catheter. The neurologic examination was repeatedly normal; his MMSE score was 25/30. His basic ADL (Katz-scale) was
8/24; the instrumental ADL score (Lawton) was 15/27. He was mobile with a four-wheel walker under supervision from his wife. He was a previous smoker and admitted drinking three alcohol consumptions per day on average. His non-fasting glycemia was $107 \mathrm{mg} / \mathrm{dL}$ (fasting reference values $70-100 \mathrm{mg} / \mathrm{dL}$ ) and follow-up revealed neither hyper- nor hypoglycemia; his hemoglobinemia was $12.6 \mathrm{~g} / \mathrm{dL}$ (reference values: 13.0- $16.5 \mathrm{~g} / \mathrm{dL}$ ).

$\mathrm{OH}$ had been documented since approximately three years, with a systolic BP varying between 73 and 93 $\mathrm{mmHg}$. An orthostatic test, two years before the present admission, had shown a BP of $184 / 111 \mathrm{mmHg}$ and a heart rate (HR) of $73 / \mathrm{min}$. in supine position. After standing one minute, BP was $98 / 70 \mathrm{mmHg}$ and pulse rate $85 / \mathrm{min}$; after three minutes $99 / 71 \mathrm{mmHg}$ and $82 /$ min.; after five minutes $98 / 63 \mathrm{mmHg}$ and $78 / \mathrm{min}$. He presented almost weekly syncopes and falls, mainly occurring after meals or physical effort. Convulsions, lasting \pm 30 seconds, had been observed once. His medical history mentioned atrial fibrillation (with periods of slow ventricular response), bronchiectasis, colon diverticulosis, urge incontinence and urine retention, treated by trans-urethral catheter, and bilateral hip prosthesis for osteoporotic fractures after falls. Three months earlier, during a hospitalization for $\mathrm{OH}(86 / 53$ $\mathrm{mmHg}$ ), a discrete rigidity of the right arm and a slight bilateral tremor of the hands had been noted, suggesting an essential tremor or a beginning PD. It had been concluded that he suffered from severe $\mathrm{OH}$, mainly due to sodium and fluid deficit, resulting in cerebral hypoperfusion. Several investigations had already been completed recently. A transthoracic cardiac ultrasound examination had shown no signs of cardiac amyloidosis, and a primary adrenal cortical failure was excluded.

His medication upon arrival consisted of rivaroxaban $15 \mathrm{mg}$ q.d., amiodarone $200 \mathrm{mg}$ q.d., bumetanide $1 \mathrm{mg}$ q.d., spironolactone $25 \mathrm{mg}$ q.d., fludrocortisone $0.1 \mathrm{mg}$ b.i.d., finasteride $5 \mathrm{mg}$ q.d., folic acid $4 \mathrm{mg}$ q.d., calcium carbonate $1000 \mathrm{mg}$ q.d., cholecalciferol 800 IE q.d., zolendronate $5 \mathrm{mg}$ i.v. once a year, and paracetamol $1 \mathrm{~g}$ if necessary. He had been advised to wear elastic stockings, rise slowly, apply a mild anti-Trendelenburg position during the night, avoid large meals, and drink sufficiently.

Upon admission to the geriatric ward amiodarone and finasteride were stopped. He developed a pneumonia, empirically treated with piperacilline and tazobactam. This treatment was successful, but he developed heart failure, necessitating fludrocortisone to be stopped.

A CT scan of the brain showed slight corticosubcortical and cerebellar atrophy, and some small lacunar infarctions in the basal ganglia. An EEG was normal. Given the history of syncopes, hypotension, and bradycardia, the cardiologist advised a coronaroangiography 
that showed only a moderate stenosis at two sites. An ultrasound examination of the neck showed no arterial stenosis, but revealed a multinodular thyroid goiter (thyroid hormone levels were found to be normal). An electromyography showed signs of length-dependent chronic axonal sensory-motoric polyneuropathy.

He had a calculated MDRD of $40 \mathrm{ml} / \mathrm{min}$, with normal renal ultrasound examination and no signs of renal artery stenosis. Continuous ECG registration confirmed a regular sinus rhythm with an average frequency of 52/ min, (33-61/min) and a second degree A-V block Mobitz type II. Placing of a pacemaker normalized the heart rhythm, but syncopes and daytime $\mathrm{OH}$ persisted.

24-hours BP registration revealed a reversal of the day-night rhythm with hypotension during the day and hypertension during the night (see Fig. 1). The daytime average systolic, diastolic, and mean arterial pressures (StDev) were respectively 98 (33.7), 59 (19.2), and 72 (24.0) $\mathrm{mm} \mathrm{Hg}$; during the night these values were respectively 147 (17.0), 85 (8.8), and 106 (11.4) mm Hg. Trying to control the BP, bumetanide and spironolactone were stopped, and fludrocortisone $(0.5 \mathrm{mg})$ was given in the morning and captopril $(12.5 \mathrm{mg})$ in the evening. With this treatment, BP values varied between 80 and 180 mmHg with a tendency for improvement towards the end of his hospitalization that continued afterwards.

A PET brain scan showed no arguments for Lewy body disease. An 18 FDG PET CT brain scan demonstrated cerebellar hypometabolism, arguing in favour of MSA.

Due to the 2020 Covid 19 pandemic, we could not realize a follow-up $24 \mathrm{~h}$ BP registration. However, the patient reported a clear improvement with a significant reduction of symptomatic events.

\section{Discussion and conclusions}

This geriatric patient with multiple morbidities, presented with heart failure, persistent $\mathrm{OH}$, and frequent falls. He had no anemia, nor diabetes. Clearly, there was a therapeutic dilemma: the need to decrease the cardiac load, resulting in $\mathrm{Na}$ loss and insufficient circulating volume on the one hand, but on the other hand the need to raise his BP.

A remarkable feature in our patient was the association of daytime $\mathrm{OH}$ with NHT. Persistent NHT has been recognized in hypertensive older patients [29]. As detailed in the background section above, the combination of $\mathrm{OH}$ and NHT is suggestive of AF, in which sympathetic activity is insufficient and provokes $\mathrm{OH}$, but where a residue of sympathetic activity often leads to hypertension in supine position and to NHT.

$\mathrm{OH}$ and NHT are more common in synucleotide diseases. Repeated neurological evaluation had not revealed PD in our patient and there were no arguments for Lewy body disease. Therefore, from a clinical point of view, he could be placed in the category of PAF. His history mentioned once the possibility of an essential tremor. Older patients presenting essential tremor do not show more AF [30], while in patients with restless legs syndrome, occurring in the context of PD, supine hypertension and NHT are more frequent [31]. Disturbed circadian BP rhythm with non-dipping has also been described in obstructive sleep apnea [32]; in our patient, a more detailed sleep observation has not been performed.

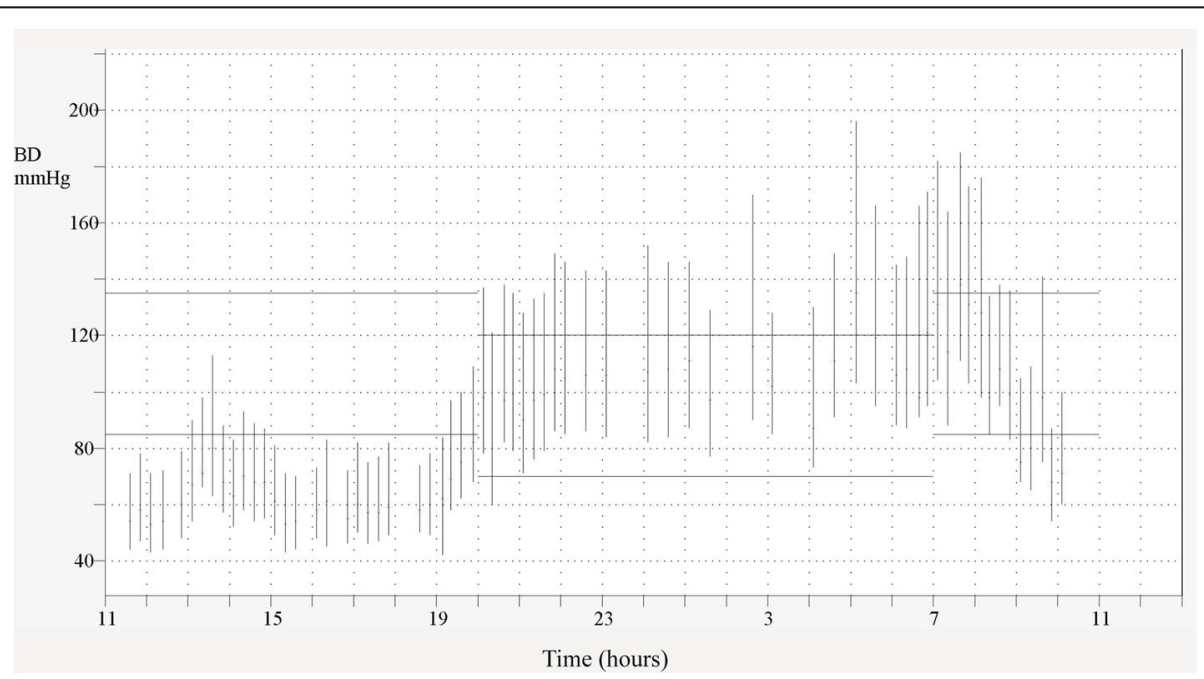

Fig. 1 Circadian measurements of arterial blood pressure at the level of the upper arm (90217A Spacelabs Healthcare). Measurements every 15 minutes from $06 \mathrm{~h}-22 \mathrm{~h}$; every 30 minutes from $22 \mathrm{~h}-06 \mathrm{~h}$. Number of measurements performed: 91; successful measurements: 67 (86\%); number of day-time (07-20 h) measurements: 42; number of night-time (patient supine, 20-07 h) measurements: 25 . Horizontal bars indicate upper and lower limits of normal values: 135 and $85 \mathrm{~mm} \mathrm{Hg}$ (day-time); 120 and $70 \mathrm{~mm} \mathrm{Hg}$ (night-time), respectively systolic and diastolic 
Besides $\mathrm{OH}$, AF often implicates other aspects, including bladder dysfunction (both incontinence and retention, as in our patient), erectile dysfunction, xerostomia, pupillary abnormalities and dry eyes, transpiration, and gastro-intestinal complaints. Other aspects may be involved, as recent basic research in mice has shown that orthosympathetic denervation of the spleen leads to disturbed immunity, and that this pathway is under control of the amygdala and the paraventricular nucleus, and is linked to behavior [33]. Hence, the complete spectrum of consequences of AF remains to be further investigated.

The orthostatic test criteria to identify $\mathrm{OH}$ remain valid for geriatric patients: $\geq 20 \mathrm{mmHg}$ systolic, or $\geq 10$ $\mathrm{mmHg}$ diastolic BP decrease within five minutes of standing, after a supine rest of five minutes [34]. Often, the expected rise in heart rate will be blunted. In $\mathrm{OH}$ patients, NHT was found to be associated (in $65 \%$ ) with an insufficient heart rhythm increase upon standing [20]. Recently, the Autonomic Disorders Consortium reported a value lower than $0.492 \mathrm{bpm} / \mathrm{mmHg}$ in the ratio $\Delta$ HeartR/ $\Delta$ Systolic BP as diagnostic to identify neurogenic $\mathrm{OH}$, with a sensitivity $91.3 \%$ and a specificity $88.4 \%$ ) [12]. In our patient, the ratio $\Delta$ HeartR/ $\Delta$ Systolic BP was 0.140 after 1 minute, 0.106 after 3 minutes, and 0.058 after 5 minutes of standing, confirming the usefulness of this ratio.

Valsalva manoeuver or the head-up tilt test are not common practice for ambulatory geriatric patients [35]. NHT is more difficult to observe and requires ambulatory $\mathrm{BP}$ monitoring with nocturnal registration.

Additional testing for AF includes 18-FDG PET CT, which in case of MSA will show cerebellar hypometabolism, as in our patient [36]. Therefore, rather than the first hypothesis of PAF, we can conclude that our patient presents MSA, despite the absence of obvious clinical neurological symptoms. In the differential diagnosis procedure, electromyography can document peripheral neuropathy, which was also present in our patient, and may have contributed to the severity of his problem. Evaluation of the cardiac and cerebral circulation is useful, as $\mathrm{OH}$ can result in impaired organ perfusion.

Treatment for $\mathrm{OH}$ will mainly consist of measures intended to alleviate BP falls, similar to those followed by our patient: wearing elastic stockings, rising slowly, avoid large meals, and drink sufficiently. Applying mild anti-Trendelenburg position will not be advisable in case of NHT. Pharmaceutical treatment will mainly consist of fludrocortisone, but NHT might be worsened. NHT is reported to react to transdermal nitroglycerin ( 0.025 to $0.1 \mathrm{mg} / \mathrm{h}$ ), applied in the evening [37]. In our patient nighttime antihypertensive treatment was preferred. A weakness in our case report is that no follow-up $24 \mathrm{~h} \mathrm{BP}$ record is available to better document the treatment effect. In mice, and still at the experimental level, treatment with a sodium-glucose cotransporter 2 (SGLT2) inhibitor has shown to normalize circadian BP rhythm $[38,39]$. To our knowledge no human studies have been performed.

In the prevention and treatment of $\mathrm{OH}$ for geriatrics patients there has been insufficient focus on intensifying muscle activity. Contracting leg muscles exert a pump function and strengthen the venous return. Intensive activity also leads to physiologic changes that upgrade homeostasis [40].

With this case report, we draw the attention to the complex situation of AF that results in simultaneous $\mathrm{OH}$ and NHT. The diagnostic approach is rather complicated for geriatric patients, as it needs continuous BP registration and cerebral imaging. Presently, preventive and therapeutic options have not been well investigated. Therefore, further studies are needed.

\section{Abbreviations \\ AF: Autonomic failure; BP: Blood pressure; CVD: Cardiovascular disease; HRV: Heart rate variability; NHT: Nocturnal hypertension; OH: Orthostatic hypotension; PAF: Pure autonomic failure; MSA: Multisystem atrophy}

\section{Acknowledgements}

Not applicable.

\section{Authors' contributions}

$S L, A D, B B$, and TM contributed in preparing the manuscript and reviewing the literature. SL and TM prepared the figures and edited the report. $S L, A D$, $B B$, and TM read and approved the final manuscript.

\section{Funding}

The authors received no funding for publication of this article.

Availability of data and materials

All data generated or analysed during this study are included in this published article [and its supplementary information files].

Ethics approval and consent to participate

This is a case report and there is no need for the ethics committee approval.

\section{Consent for publication}

The patient gave written consent to use anonymized imaging and clinical information for publication.

\section{Competing interests}

The authors declare that they have no competing interests.

\section{Author details}

'Department of Geriatrics, Universitair Ziekenhuis Brussel, Laarbeeklaan 101, B-1090 Brussels, Belgium. 'Frailty in Aging (FRIA) investigation group, Faculty of Medicine \& Pharmacy, Vrije Universiteit Brussel (VUB), Laarbeeklaan 103, B-1090 Brussels, Belgium. ${ }^{3}$ Departments of Endocrinology \& Clinical Pharmacology, Universitair Ziekenhuis Brussel, Laarbeeklaan 101, B-1090 Brussels, Belgium.

Received: 20 August 2020 Accepted: 1 February 2021

Published online: 01 March 2021

References

1. Finucane C, O'Connell MDL, Donoghue O, et al. Impaired Orthostatic Blood Pressure Recovery Is Associated with Unexplained and Injurious Falls. J Am Geriatr Soc. 2017;65:474-82. https://doi.org/10.1111/jgs.14563. 
2. Rutan $\mathrm{GH}$, Hermanson $\mathrm{B}$, Bild DE, et al. Orthostatic hypotension in older adults. The Cardiovascular Health Study. CHS Collaborative Research Group. Hypertension. 1992;19(6 Pt 1):508-19. https://doi.org/10.1161/01.hyp.19.6.508.

3. Huang CC, Sandroni P, Sletten DM, et al. Effect of age on adrenergic and vagal baroreflex sensitivity in normal subjects. Muscle Nerve. 2007;36:637 42. https://doi.org/10.1002/mus.20853.

4. Jansen RW, Connelly CM, Kelley-Gagnon MM, Parker JA, Lipsitz LA. Postprandial hypotension in elderly patients with unexplained syncope. Arch Intern Med. 1995;155:945-52.

5. Robertson D, Robertson RM. Causes of chronic orthostatic hypotension. Arch Intern Med. 1994;154:1620-4.

6. Paul J, Vaillant F, Vanden Bossche $\mathrm{O}$, et al. Orthostatic blood pressure drop and cardiovascular and psychotropic medication dosages in acutely ill geriatric inpatients. Acta Clin Belg. 2019;29:1-8. https://doi.org/10.1080/1 7843286.2019.1623516.

7. Saladini F, Attilio Di Marco A, Paolo Palatini P. Autonomic Dysfunction: How to Identify and When to Treat? High Blood Press Cardiovasc Prev. 2016;23: 237-43. https://doi.org/10.1007/s40292-016-0162-3.

8. Consensus statement on the definition of orthostatic. hypotension, pure autonomic failure, and multiple system atrophy. The Consensus Committee of the American Autonomic Society and the American Academy of Neurology. Neurology. 1996;46:1470. https:/doi.org/10.1212/wnl.46.5.1470.

9. Bäckman L, Nyberg $L$, Lindenberger $U$, et al. The correlative triad among aging, dopamine, and cognition: current status and future prospects. Neurosci Biobehav Rev. 2006;30:791-807. https://doi.org/10.1016/j. neubiorev.2006.06.005.

10. Yuan J, Chang SY, Yin SG, et al. Two conserved epigenetic regulators prevent healthy ageing. Nature. 2020;579:118-22. https://doi.org/10.1038/ s41586-020-2037-y.

11. Norcliffe-Kaufmann L, Kaufmann H, Palma JA, et al. Autonomic Disorders Consortium. Orthostatic heart rate changes in patients with autonomic failure caused by neurodegenerative synucleinopathies. Ann Neurol. 2018; 83:522-31. https://doi.org/10.1002/ana.25170.

12. Moore A, Ryan J, Watts $\mathrm{M}$, et al. Orthostatic tolerance in older patients with vitamin B12 deficiency before and after vitamin B12 replacement. Clin Auton Res. 2004;14:67-71. https://doi.org/10.1007/s10286-004-0142-x.

13. Vinik Al, Ziegler D. Diabetic Cardiovascular Autonomic Neuropathy. Circulation. 2007:115:387-97. https://doi.org/10.1161/CIRCULATIONAHA.106. 634949.

14. Salman IM. Cardiovascular Autonomic Dysfunction in Chronic Kidney Disease: a Comprehensive Review. Curr Hypertens Rep. 2015;17:59. DOI https://doi.org/10.1007/s11906-015-0571-z.

15. Williams B, Mancia G, Spiering W, et al. 2018 ESC/ESH Guidelines for the management of arterial hypertension. Eur Heart J. 2018 Sep 1;39(33):30213104. https://doi.org/10.1093/eurheartj/ehy339.

16. Mann S, Altman DG, Raftery EB, Bannister R. Circadian variation of blood pressure in autonomic failure. Circulation. 1983;68:477-83. https://doi.org/1 0.1161/01.cir.68.3.477.

17. Goldstein DS, Pechnik S, Holmes C, et al. Association between supine hypertension and orthostatic hypotension in autonomic failure. Hypertension. 2003;42:136-42. https://doi.org/10.1161/01.HYP.0000081216.11 623.C3.

18. Shannon JR, Jordan J, Diedrich A, et al. Sympathetically mediated hypertension in autonomic failure. Circulation. 2000;101:2710-5. https://doi. org/10.1161/01.cir.101.23.2710.

19. Chisholm P, Anpalahan M. Orthostatic hypotension - pathophysiology, assessment, treatment, and the paradox of supine hypertension. Intern Med J. 2017:47:370-9. https://doi.org/10.1111/imj.13171.

20. Anpalahan A, Anpalahan M. Nocturnal hypertension and abnormal circadian blood pressure patterns in orthostatic hypotension. Intern Med J. 2019;49: 1191-2. https://doi.org/10.1111/imj.14425.

21. Berganzo K, Díez-Arrola B, Tijero B, Somme J, et al. Nocturnal hypertension and dysautonomia in patients with Parkinson's disease: are they related? J Neurol. 2013;260:1752-6. https://doi.org/10.1007/s00415-013-6859-5.

22. Kim JS, Lee SH, Oh YS, et al. Cardiovascular Autonomic Dysfunction in Mild and Advanced Parkinson's Disease. J Mov Disord. 2016;9:97-103. https://doi. org/10.14802/jmd.16001.

23. Tsioufis C, Antoniadis D, Stefanadis C, et al. Relationships between new risk factors and circadian blood pressure variation in untreated subjects with essential hypertension. Am J Hypertens. 2002;15:600-4. https://doi.org/10.1 016/s0895-7061(02)02954-0.
24. Cicconetti P, Morelli S, Ottaviani L, et al. Blunted nocturnal fall in blood pressure and left ventricular mass in elderly individuals with recently diagnosed isolated systolic hypertension. Am J Hypertens. 2003;16:900-5. https://doi.org/10.1016/s0895-7061(03)01012-4.

25. Karaagac K, Vatansever F, Tenekecioglu E, et al. The Relationship between Non-Dipper Blood Pressure and Thoracic Aortic Diameter in Metabolic Syndrome. Eurasian J Med. 2014;46:120-5. https://doi.org/10. 5152/eajm.2014.27.

26. Fedecostante M, Spannella F, Cola G, et al. Chronic Kidney Disease Is Characterized by "Double Trouble" Higher Pulse Pressure plus Night-Time Systolic Blood Pressure and More Severe Cardiac Damage. PLoS One. 2014; 9:e86155. https://doi.org/10.1371/journal.pone.0086155.

27. Wolters FJ, Mattace-Raso FUS, Koudstaal PJ, et al. Orthostatic Hypotension and the Long-Term Risk of Dementia: A Population-Based Study. PLoS Med. 2016;13:e1002143. https://doi.org/10.1371/journal.pmed.1002143.

28. Guo $H$, Tabara $Y$, Igase $M$, et al. Abnormal nocturnal blood pressure profile is associated with mild cognitive impairment in the elderly: the J-SHIPP study. Hypertens Res. 2010;33:32-6. https://doi.org/10.1038/hr.2009.172.

29. Kobrin MD, Oligman W, Kumar A, et al. Diurnal variation of blood pressure in elderly patients with essential hypertension. J Am Geriat Soc. 1984;32: 896-9. https://doi.org/10.1111/j.1532-5415.1984.tb00890.x.

30. Kim JS, Oh YS, Park HE, et al. Cardiovascular autonomic dysfunctions in elderly patients with essential tremor: comparison with healthy controls. Neurol Sci. 2016;37:711-6. https://doi.org/10.1007/s10072-015-2465-X.

31. Oh YS, Kim JS, Park IS, et al. Association between nocturnal/supine hypertension and restless legs syndrome in patients with Parkinson's disease. J Neurol Sci. 2014;344:186-9. https://doi.org/10.1016/j.jns.2014. 06.056 .

32. Kanbay M, Turgut F, Uyar ME, et al. Causes and Mechanisms of Nondipping Hypertension. Clin Exp Hypertens. 2008;30:585-97. https://doi.org/10.1080/1 0641960802251974.

33. Zhang $X$, Lei $B$, Yuan $Y$, et al. Brain control of humoral immune responses amenable to behavioural modulation. Nature. 2020;581:204-8. https://doi. org/10.1038/s41586-020-2235-7.

34. Soysal P, Aydin AE, Okudur SK, Isik AT. When should orthostatic blood pressure changes be evaluated in elderly: 1st, 3rd or 5th minute? Arch Gerontol Geriatr. 2016;65:199-203. https://doi.org/10.1016/j.archger.2016. 03.022 .

35. Zygmunt A, Stanczyk J. Methods of evaluation of autonomic nervous system function. Arch Med Sci. 2010;6:11-8. https://doi.org/10.5114/aoms.2 010.13500.

36. Brooks DJ, Seppi K. Neuroimaging Working Group on MSA. Proposed neuroimaging criteria for the diagnosis of multiple system atrophy. Mov Disord. 2009;24:949-64. https://doi.org/10.1002/mds.22413.

37. Shannon JR, Jordan J, Costa F, et al. The hypertension of autonomic failure and its treatment. Hypertension. 1997;30:1062-7. https://doi.org/10.1161/01. hyp.30.5.1062.

38. Rahman A, Hitomi H, Nishiyama A. Cardioprotective Effects of SGLT2 Inhibitors Are Possibly Associated With Normalization of the Circadian Rhythm of Blood Pressure. Hypertens Res. 2017;40:535-40. https://doi.org/1 0.1038/hr.2016.193.

39. Wan N, Rahman A, Hitomi H, Nishiyama A. The Effects of SodiumGlucose Cotransporter 2 Inhibitors on Sympathetic Nervous Activity. Front Endocrinol (Lausanne). 2018;9:421. https://doi.org/10.3389/fendo.2 018.00421.

40. Carroll JF, Wood CE, Pollock ML, et al. Hormonal responses in elders experiencing pre-syncopal symptoms during head-up tilt before and after exercise training. J Gerontol A Biol Sci Med Sci. 1995;50:M324-9. https://doi. org/10.1093/gerona/50a.6.m324.

\section{Publisher's Note}

Springer Nature remains neutral with regard to jurisdictional claims in published maps and institutional affiliations. 